\title{
Several Populations Genetic Algorithms
}

\author{
Chunyan Liao \\ (School of Foreign Languages, Wuhan Textile University, Wuhan Hubei 430073, China) \\ cinderellaandfish@126.com
}

\begin{abstract}
In the traditional genetic algorithm that the convergence of the existence of slow convergence and local convergence problems, the introduction of a variety of groups based on the standard genetic algorithm to overcome the premature convergence of genetic algorithms. After taking into account the evolution of genetic fitness of the individual problems in the algorithm used in populations of competitive methods, in specific populations to adapt to constantly out of the individual is low, and continue to add new individuals to increase the diversity of the population in order to improve convergence speed. Test function of a typical experiment, the results with other multi-group comparison of the results of genetic algorithm, the results demonstrate that the new algorithm is superior in avoiding premature convergence to find high-quality solutions.
\end{abstract}

Keywords-Several populations; genetic algorithm; improved algorithm; prematurity

\section{INTRODUCTION}

The origin of genetic algorithem is the $r$ esearch of biology using computer. The algorithm is proposed by professor Holland in 1975, it is the optimize algorithm based on darwinian evolution. The working process of the algorithm is: 1 a group is generated with certain of chromosome; 2 a function that used to judge the better or worse of the chromosome, do the jo b of select, crossover and mutation, which can get a new p opulation, repeat the operation and get the best result[1].

The genetic algorithm supply a framework that used to get the complex problems, the characters is the process is not depend on the domain and the genetic algorithm is heuristic way. The simple genetic algorithm has been us ed in Combinatorial Optimization, intelligent control, image processing and so on. The simple genetic algorithm is simple and easy operating, but the gener ation, select, crossover and mutation of the population is random, which may be cause the premature or slow convergence in global and cannot get the $b$ est result[2]. There many people propose al ot of schema to improve algorithm, these improvements are used to i mprove the way to select, crossover, mutation and the fitness[3,4]. All these improvement have the limitations in efficiency of operating and the velocity of convergence.

In order to overcome the premature of the genetic algorithm, in this paper we proposed a genetic algorithm based on evolution, which can assure the best individuals to be retained. The algorithm can i mprove the operating efficiency [5], with the exp eriments, the algorithm can improve the velocity of evolution and can get best result in global.

\section{THE PROBLEM OF THE GENETIC ALGORITHM}

\section{A Premature Convergence}

In genetic algorithm, there are some exceptions. 1 The premature convergence of a genetic algorithm arises when the genes of some high rated Individuals quickly attain to dominate the population, constraining it to co nverge to a local optimum. In this case, the genetic operators cannot produce any more descendents better that the parents. 2 when the fitness of the individuals are nearly, the diversity of the population are reduced, which will reduce the chance of producing the new individuals. A new w ay of judge the premature degree is proposed as follows [6]:

Definiton 1: assume the scope of the population (marked as $\mathrm{P}$ )is $\mathrm{n}, \quad P=\left\{a_{1}, a_{2}, \cdots a_{n}\right\}$, and $a_{j}=\left(a_{1 j}, a_{2 j}, \cdots a_{l j}\right)$, the fitness is $f\left(a_{j}\right)$, the average fitness is $f_{\text {avg }}=\frac{1}{n} \sum_{j=1}^{n} f\left(a_{j}\right)$.the b est fitness is $f_{\max }$. $\overline{f_{\max }}$ is the average fitness that larger than the $f_{\text {avg }}$. We defined the difference of the $f_{\max }$ and $\overline{f_{\max }}$ ( $\varepsilon=f_{\text {max }}-\overline{f_{\text {max }}}$ ) is the degree of the premature.

\section{B Local Search Problems}

Genetic algorithm is good at searching in global, not in searching in local, which will lead to reduce the velocity of convergence, even cannot get the best result in global.

\section{Non-Directional of the Genetic Factors}

The genetic factors can a ssure the chosen factors are individuals. The mutagenic factors and interleave factor will generate a new individual, but do not care the whether the individual is better. If the new individual is not better, the new individual is interference factor and will reduce the velocity of evolution.

\section{DIVERSE GENETIC ALGORITHMS}

Diverse genetic algorithm apply the para llel genetic algorithm on simple genetic algorithm. The algorithm is: for 
the problem, the sub populations are $g$ enerated and the scope is $\mathrm{N}$, and there are $\mathrm{n}$ i ndividuals. The gen etic algorithms run on different sub populations respectively. In some situation, some information can be exchanged among the sub populations. The algorithm of the sub populations should be different and the results of the algorithms are processed at last, which lead to $s$ atisfy the $r$ equest. The multiple-populations model not only can av oid the pre-convergence, but also can improve the performance of the genetic algorithm and the velocity of evolution. In this paper, we improve the algorithm based on the parallel genetic algorithm.

\section{A Local Competition of the populations}

When do the job of crossover and mutation, the fitness degree after the evolution may be reduce, the population may be lost the best individual. We proposed a sche malocal competition: the s chema checks whether the child-population is s imilarly to the parent-population after the individual in the sub population do the job of crossover and mutation, at the sa me time, the fitness degree is considered, the lager will remain as the $\mathrm{c}$ hild-population. The schema is better the traditional way, which remain the best individual and the best in dividual participated the crossover. The schema gives chance to $i$ mprove the individual and better for getting the best result. The operation of mutation is same to the crossover, the operated individual should be compared to pre-operate one, when the fitness of the operated individual is larger than the pre-operate one; the individual is select ed as the next generation, on the other hand, the pre-operate individual is selected. This way will avoid the better individuals to lost and make the better individuals participating the crossover and mutation.

\section{B Harmonic Average Fitness}

The average fitness refect the average quality of the popution, which can not asure that the next generation contain the better result than the parent generation. The best fitness degree can reflect the quality of the individual, but can not reflect the quality of the whole populaiton. In order to compare the quality of the sub population, we proposed the harmonic average fitness of the population[7]

Definition 2. evolution algebra is $\mathrm{T}$, the $\mathrm{t}^{\text {th }}$ population composed by $x_{t}^{1}, x_{t}^{2}, \cdots x_{t}^{n}$, and the fitness is $f_{t}^{1}, f_{t}^{2}, \cdots, f_{t}^{n}$. The average fitness of the individual is $f_{\text {avg }}, \max$ fitness $f_{t \text { max }}=\max \left\{f_{t}^{i}, i=1,2, \cdots n\right\}$, then we get the harmonic average fitness $\overline{f_{t}}=f_{\text {avg }} \times\left(1-\frac{t}{T}\right)+f_{t \cdot \max } \times \frac{t}{T}$

The harmonic average fitness takes the average fitness and the max fitness into coun t. In early evol ution, the average fitness plays the main role and assures the global evolution; at later evolution, the max fitness play main role and assure the key evolution.

\section{Condition of Evolution Stop}

The stop of the evolution is controled by two conditions, the generations of the evolution and the cong ergence conditions. The convergence of the populaion is the schema that when the fitness of the $(t+1)^{\text {th }}$ generation is not better than the fitness of the $\mathrm{t}^{\text {th }}$ generation, the algorithm over.

\section{$D$ The Step of the Algorithm}

The step of the diverse genetic algorithms is discribed as fellows:

Step $1 \mathrm{n}$ in dividuals are chosed from the whole population, the individuals are divided into 3 same populations, marked as $\mathrm{P}_{1}[\mathrm{k}] 、 \mathrm{P}_{2}[\mathrm{k}] 、 \mathrm{P}_{3}[\mathrm{k}]$. The binary coding is good at search and suit to be process by cpu. In this paper, the binary is used to coding.

Step 2 computing the fitness of the first generation;

Step 3 playing the job of select, crossover and mutation on very population. The probab ility of the crossover and mutation are defined by us, th e select $f$ actors is the traditional genetic algorithm, roulette. The individual with larger fitness will be selected, and with the high probability to enter next generation.

Step 4 the fitness of individuals in the population are computed, the top 4 individuals are selected and a new population is generated.

Step 5 the new population will compete with the old 3 individuals, goto step3;

Step 6 select t op 3 individuals from the 4 populations and composed a new population, which will replace the $4^{\text {th }}$ population.

Step 7 repeat the step 5 and step 6 ;

Step 8 if the best fitness in the po pulation satisfied the request, or the iterations reach the given generations, the algorithm stop, otherwise, goto step 3 .

The single crossover operation is applied to the binary coding. The characters of the single crossover are: if relations of the adjacent genes can sup ply the better individual fitness, the o peration will have $\mathrm{t}$ he low probability of reducing the whole fitness. The algorithm is different from the traditional algorithm is: 1 increasing the number of the init individuals, which increasing the searching scope; 2 deleting the similarity individuals, increasing the diversities of $p$ opulation, which avoid the pre-convergence and the best result in local.

\section{TEST OF THE ALGORITHM}

\section{A Descriptions of the Test}

We introduced the Dejong's target function[8], function F1 is :

$$
\left\{\begin{array}{l}
f(x)=\sum_{i=1}^{3} x_{i}^{2} \\
-5.12 \leq x_{i} \leq 5.12 ; i=1,2,3
\end{array}\right.
$$

Function $\mathrm{F} 1$ is a function of single peak value, which always used to test the result and the efficiency of the optimizing, obviously, the function will get the max value 
in endpoint, $\max f(x)=78.6432$. There are many optimizing result of the genetic algorithm[9], the best on e is the new fuzzy genetic algorithm, which can make the result approach to 78.64 within 54 generation. Paper[11] proposed a multi population algorithm based on entropy, which using the space co nvergence and can get the best result $\mathrm{F}(\mathrm{x})=78.640630$ in 21 generations, at the same time, $\mathrm{F} 1$ can get the min value in global, but cannot convergence in min point.

$$
\left\{\begin{array}{l}
f\left(x_{1}, x_{2}\right)=100\left(x_{i}^{2}-x_{2}\right)^{2}+\left(1-x_{1}\right)^{2} \\
-2.048 \leq x_{i} \leq 2.048 ; i=1,2
\end{array}\right.
$$

$\mathrm{F} 2$ is used to get the max value of the Rosenbrock in global, there are two $\max$ value in local: $F(2.048,-2.048)=3$ 897.7342 and $F(-2.048,-2.048)=3905.9262$, the latter is the max value in gl obal. Paper[10] proposed a s chema based on random perturbation, which can get the best result in 100 generations. In paper[11], a schema can get the best result $F(x)=3905.243$ in 10 generations, at the same time, there is a min value $\mathrm{F}(0,0)=0$, but it is ab normal and not easy to get the min value in global.

\section{$B$ The Test Result and Analysis}

We used the matlab7.0 to emulate the algorithm. In order to eliminate the special effect, we run the algorithm many times. When test the function, we applied the binary coding, single crossover and single mutation, the rate of the crossover is 0.7 and the rate of mutation is 0.1 . The i nit scale of population is 20 , the number of population is 4 .

Figure 1-4 give the evolvement of the F1 and F2, figure 1 and figure 2 used the min fitness to evolve, figure 3 and figure 4 used the max fitness to evolve, table 1 and table 2 give the compare between ours' schema and others.

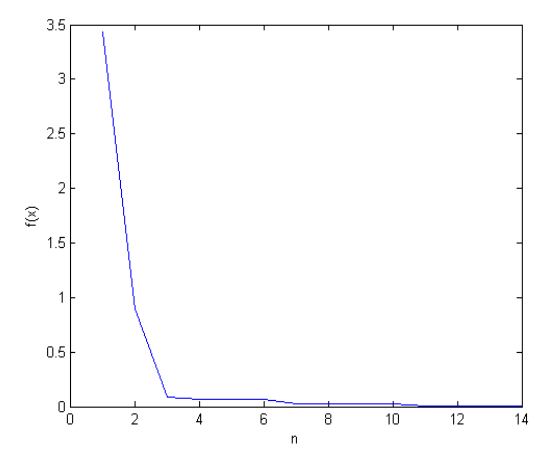

FIGURE 1 THE GRAPH OF F1 WITH MIN FITNESS

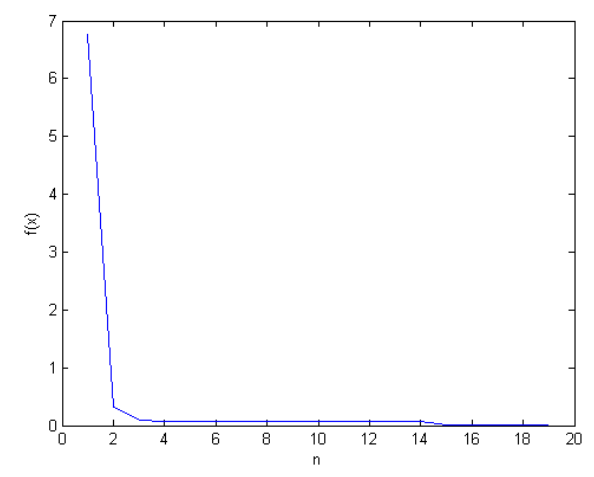

FIGURE 2 GRAPH OF F2 WITH MIN FITNESS

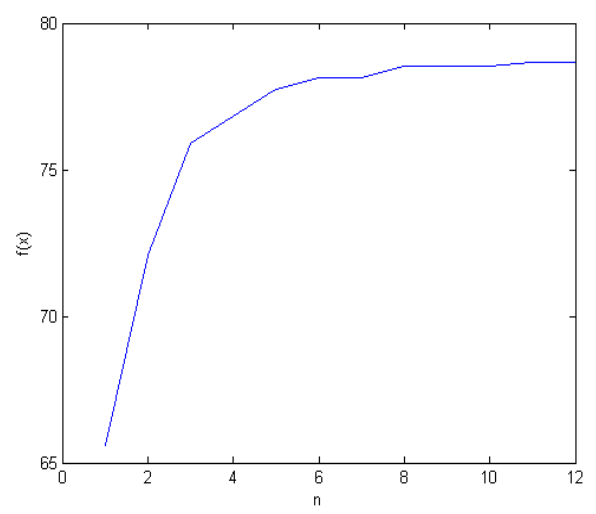

FIGURE 3 GRAPH OF F1 WITH MAX FITNESS 


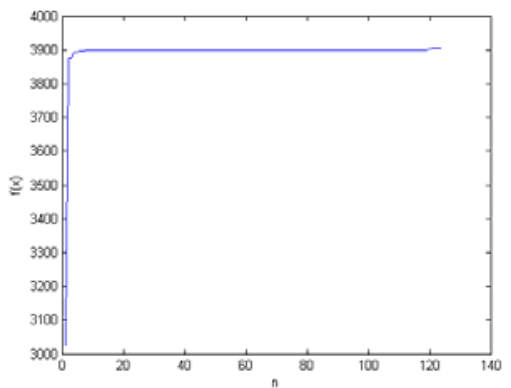

FIGURE 4 GRAPH OF F2 WITH MAX FITNESS

TABLE 1 COMPARE OF OPTIMIZATION WITH MIN FITNESS

\begin{tabular}{|l|l|l|l|}
\hline & Way of genetic & Generations & Best value \\
\hline F1 & Multi population & 14 & 0.00748 \\
\hline & Single population & 18 & 0.00752 \\
\hline F2 & Multi population & 19 & 0.000005 \\
\hline & Single population & 21 & 0.003807 \\
\hline
\end{tabular}

TABLE 2 COMPARE OF OPTIMIZATION MAX FITNESS

\begin{tabular}{|c|c|c|c|c|c|c|c|}
\hline \multirow[t]{2}{*}{ Function } & \multirow{2}{*}{$\begin{array}{l}\text { Best value } \\
\text { in theory }\end{array}$} & \multicolumn{3}{|c|}{ Result of our schema } & \multicolumn{3}{|c|}{ Result of others } \\
\hline & & $\begin{array}{l}\text { Way of } \\
\text { genetic }\end{array}$ & generations & $\begin{array}{l}\text { Best } \\
\text { value }\end{array}$ & Referenc & generations & Best value \\
\hline \multirow[t]{3}{*}{ F1 } & \multirow[t]{3}{*}{78.64325} & \multirow{2}{*}{$\begin{array}{l}\text { Multi } \\
\text { population }\end{array}$} & \multirow[t]{2}{*}{12} & \multirow[t]{2}{*}{78.6432} & [8] & 60 & 78.64 \\
\hline & & & & & [10] & 21 & 78.640 .630 \\
\hline & & Single & 18 & 78.5408 & & & \\
\hline \multirow[t]{3}{*}{$\mathrm{F} 2$} & \multirow[t]{3}{*}{3905.9262} & \multirow{2}{*}{$\begin{array}{l}\text { Multi } \\
\text { population }\end{array}$} & \multirow[t]{2}{*}{10} & \multirow[t]{2}{*}{3905.926} & [9] & 100 & 3905.909 \\
\hline & & & & & {$[10]$} & 10 & 3905.243 \\
\hline & & Single & 25 & 3897.733 & & & \\
\hline
\end{tabular}

With the table 1 and table 2 , the genetic algorithm with single population has the shortcomings, premature and pre-convergence. The improved schema can suppress the premature and pre-convergence, and the precision of convergence will improved.

\section{FUTURE WORKS}

In order to resolv e the problem of premature and the time consume, we proposed a harmonic average fitness to judge the $b$ etter or worse of the population, which can improve the efficiency of the genetic, at the same time, the schema can avoid the premature. The algorithm can assure the best optimization result and reduce the process of the genetic algorithm. With experiments, the schema is stably, reliable and good at searching in global, the schema improved convergence velocity. We will do more jobs on the improvement of the genetic algorithm.

\section{REFERENCE}

[1]Holland JH. Adaptation in national and arti ficial systems[M].Michiban:The University of Michigan Press, 1975.

[2]YE Zai-fu, SHAN Yuan-da. A new transmission network expansion planning based on multiple-population genetic algorithm[J]. Automation of electric power systems. 2000: 24 -35
[3] Gong tun wei,Sun xiaoyan,Guo xijin. Novel survival of the fittest genetic algorithm [J],Control and decision , 2002,11(6):908-912.

[4] Han wanlin.Improvement of Genetic Algorithm[J], Journal of CUMT,2001,3(1):102-105.

[5] Chen changzheng,Wang lang,Adaptive Selection of Crossover and Mutation Probability of Genetic Algorithm and Its Mechanism[J], Control Theory \& Applications, 2002,2(1):41-44.

[6]Song zefeng, Zhou wanzheng. An A ssociation Rule Mining Based on Genetic Algorithm[J]. Computing Technology and Automation,2005(8).

[7]Wang wenyi,Qin guangyi,Wang ruoyu. Research on Adaptive and Multipopulation Parallel Genetic Algorithm[J], Computer Engineering and Applications, 2006 42(15)

[8] DeJong , K.A.. The Analysis of the Behavior of a Class of Genetic Adaptive Systems.Doctoral Dissertation,University of Michigan, Michigan ,1975.

[9] Li qing, Zheng deling, Tang yong. A New Kind of Fu zzy Genetic Algorithm[J], Journal of University of Science and Technology Beijing, 2001,23(1):85-89.

[10] He xiongjun,Sun guozheng,Liu Gang. Random Perturbation Method of Genetic Algorithms[J], Journal of Wuhan University(Natural Science Edition), 2001,23(1):85-89.

[11] Li c hunlian,Wang xicheng,Zhao jincheng, An information entropy-based multi-population genetic algorithm[J], Journal of Dalian University of Technology, 2004,44(4) 